PROCEEDINGS OF THE

AMERICAN MATHEMATICAL SOCIETY

Volume 133, Number 12, Pages 3441-3445

S 0002-9939(05)07663-X

Article electronically published on July 13, 2005

\title{
BOUNDS FOR THE INDEX OF THE CENTRE IN CAPABLE GROUPS
}

\author{
K. PODOSKI AND B. SZEGEDY
}

(Communicated by Jonathan I. Hall)

\begin{abstract}
A group $H$ is called capable if it is isomorphic to $G / \mathbf{Z}(G)$ for some group $G$. Let $H$ be a capable group. I. M. Isaacs (2001) showed that if $H$ is finite, then the index of the centre is bounded above by some function of $\left|H^{\prime}\right|$. We show that if $\left|H^{\prime}\right|<\infty$, then $|H: Z(H)| \leq\left|H^{\prime}\right|^{c \log _{2}\left|H^{\prime}\right|}$ with some constant $c$ and this bound is essentially best possible. We complete a result of Isaacs, showing that if $H^{\prime}$ is a cyclic group, then $|H: \mathbf{Z}(H)| \leq\left|H^{\prime}\right|^{2}$.
\end{abstract}

\section{INTRODUCTION}

Let $G$ be an arbitrary group. According to a classical theorem of Schur, if $|G: \mathbf{Z}(G)|<\infty$, then $\left|G^{\prime}\right|<\infty$. An easy argument based on the ultra product method shows that there is a bound for the order of the derived subgroup in terms of the index of the centre. The best bound was given by Wiegold [7] showing that if $|G: \mathbf{Z}(G)|=n$, then $\left|G^{\prime}\right| \leq n^{\frac{1}{2} \log _{2} n}$. Infinite extraspecial groups show that the converse of the theorem of Schur does not hold in general. However, P. Hall (see 6, p.423) observed that if $\left|G^{\prime}\right|<\infty$, then $\left|G: \mathbf{Z}_{\mathbf{2}}(G)\right|$ is bounded above in terms of $\left|G^{\prime}\right|$ (where $\mathbf{Z}_{\mathbf{2}}(G)$ denotes the second member of the upper central series of $G$ ). The first explicit bound was given by I. D. Macdonald [3. Improving this bound we proved in [5] that

$$
\left|G: \mathbf{Z}_{\mathbf{2}}(G)\right| \leq\left|G^{\prime}\right|^{\log _{2}\left|G^{\prime}\right|},
$$

and our examples show that this estimate is sharp apart from the value of the constant $c$.

A group $H$ is said to be capable if there exists some group $G$ such that $G / \mathbf{Z}(G)$ is isomorphic to $H$. I. M. Isaacs [2 proved that if $H$ is a capable group and $\left|H^{\prime}\right|=n$, then $|H: \mathbf{Z}(H)|$ is bounded above by some function $f$ of $n$, or equivalently, if $G$ is an arbitrary group and $\left|G^{\prime}: G^{\prime} \cap \mathbf{Z}(G)\right|=n$, then $\left|G: \mathbf{Z}_{\mathbf{2}}(G)\right| \leq f(n)$. However, he has not given an explicit function $f(n)$. In our present paper we give the essentially best possible bound.

Theorem 1. If $G$ is a group (not necessarily finite) and $\left|G^{\prime}: G^{\prime} \cap \mathbf{Z}(G)\right|=n$, then $\left|G: \mathbf{Z}_{\mathbf{2}}(G)\right| \leq n^{c \log _{2} n}$ with $c=2$.

Received by the editors March 10, 2003 and, in revised form, January 6, 2004.

2000 Mathematics Subject Classification. Primary 20E34, 20D60, 20D15, 20D25.

This research was partially supported by the Hungarian National Research Foundation (OTKA), grant no. T038059. 
Using this result for $H=G / \mathbf{Z}(G)$ we obtain the following.

Corollary 2. If $H$ is a capable group and $\left|H^{\prime}\right|=n$, then

$$
|H: \mathbf{Z}(H)| \leq n^{c \log _{2} n}
$$

with $c=2$.

Actually, the preceding result can be regarded as a converse of Wiegold's theorem. The sequence of groups $G_{n}$ we constructed in [5] shows that these estimates are sharp apart from the value of the constant $c$. The proof of Theorem 1 shows that the value of the constant $c$ is at most 2. We also mention that H. Heineken [1] constructed capable groups $H$ for all odd prime numbers $p$ and for all natural

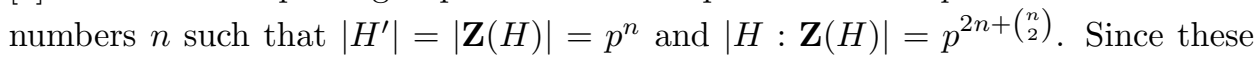
are the best known examples, we think that the constant $c$ can be further improved. Although the above examples do not work for $p=2$, similar estimates motivate us to think that perhaps $c=\frac{1}{2}$ is the best constant.

Question 3. Is it true that if $H$ is a capable group and $\left|H^{\prime}\right|=n$, then $|H: \mathbf{Z}(H)| \leq$ $n^{\frac{1}{2} \log _{2} n+c_{2}}$ for some constant $c_{2}$ ?

For groups with infinite derived subgroup a similar argument yields:

Theorem 4. If $G$ is a group and $\left|G^{\prime}: G^{\prime} \cap \mathbf{Z}(G)\right|=\kappa$ is an infinite cardinal, then $\left|G: \mathbf{Z}_{\mathbf{2}}(G)\right| \leq 2^{\kappa}$.

Corollary 5. If $H$ is a capable group and $\left|H^{\prime}\right|=\kappa$ is an infinite cardinal, then $|H: \mathbf{Z}(H)| \leq 2^{\kappa}$.

Remark 6. Related to infinite groups, similar results are included in [4] and [5]. For each infinite cardinal $\kappa$ we constructed a group $G$ such that $\left|G^{\prime}\right|=\kappa, \mathbf{Z}(G)=1$ and $|G|=2^{\kappa}$ (see [5]). It follows that the previous estimates are sharp.

The second part of our paper deals with groups with cyclic derived subgroups.

For a capable group $H$, I. M. Isaacs 2] proved that if $H$ is finite, $H^{\prime}$ is cyclic and all elements of order 4 in $H^{\prime}$ are central in $H$, then $|H: \mathbf{Z}(H)| \leq\left|H^{\prime}\right|^{2}$. In the present paper we prove that the assumption about elements of order 4 can be omitted.

Theorem 7. If $H$ is a finite capable group and $H^{\prime}$ is cyclic, then $|H: \mathbf{Z}(H)| \leq$ $\left|H^{\prime}\right|^{2}$.

For an arbitrary group $G$, we prove the following estimate.

Theorem 8. If $G$ is a finite group with $G^{\prime}$ cyclic of order $n$, then $\left|G: \mathbf{Z}_{\mathbf{2}}(G)\right| \leq$ $n \varphi(n)$, where $\varphi$ is Euler's totient function.

The previous estimate is sharp for the holomorph of a cyclic group.

\section{Groups With ARbitrary DERIVED Subgroups}

In this section we prove Theorem 1 and Theorem 4.

Lemma 9. Let $H$ be a subgroup of $G$ generated by $k$ elements and $\left|G^{\prime}\right|=n$. Then $\left|G: C_{G}(H)\right| \leq n^{k}$. 
Proof. Let $x_{1}, x_{2}, \ldots, x_{k}$ be a generating system of $H$. Let us denote the conjugacy class of $x_{i}$ in $G$ by $C l\left(x_{i}\right)$. Then

$$
\left|G: C_{G}(H)\right| \leq \prod_{i=1}^{k}\left|G: C_{G}\left(x_{i}\right)\right|=\prod_{i=1}^{k}\left|C l\left(x_{i}\right)\right| \leq\left|G^{\prime}\right|^{k}=n^{k} .
$$

Lemma 10. Let $G$ be an arbitrary group and $C<G$ be a proper subgroup. Then $G^{\prime}=[G-C, G]$.

Proof. It is enough to generate the commutators $\{[c, g] \mid c \in C ; g \in G\}$. Let $x$ be an arbitrary element of $G-C$. Then

$$
[c, g]=\left[x, c^{-1} g c\right]^{-1}[c x, g] \in[G-C, G] .
$$

Lemma 11. Let $Z=G^{\prime} \cap \mathbf{Z}(G)$, and let $U, V$ be subgroups of $G$ such that $Z \leq$ $U, V \leq G^{\prime}$. Then there exist elements $y, z$ of $G$ with the following properties.

(1) If $Z \supsetneqq U$, then $U \cap C_{G}(y) \supsetneqq U$.

(2) If $V \supsetneqq G^{\prime}$, then $V \supsetneqq\langle V,[y, z]\rangle$.

Proof. Set $C=C_{G}(U)$. Suppose that $Z \supsetneqq U$. Now, $C \supsetneqq G$; thus $U \cap C_{G}(y) \supsetneqq U$ for all $y \in G-C$. Lemma 10 yields that $G^{\prime}=[G-C, G]$. Consequently, if $V \supsetneqq G^{\prime}$, then we can choose $y \in G-C$ and $z \in G$ such that $V \supsetneqq\langle V,[y, z]\rangle$. In the case of $Z=U$ and $V \supsetneqq G^{\prime}$, then we can choose arbitrary $[y, z] \notin V$.

Lemma 12. Let $Z=G^{\prime} \cap \mathbf{Z}(G)$, and suppose that $\left|G^{\prime}: Z\right|=n$. Let $T$ be a subgroup with $G^{\prime} \leq T \leq G$ having the following properties.

(1) $G^{\prime}=T^{\prime} Z$.

(2) $G^{\prime} \cap \mathbf{Z}(T)=Z$.

(3) $T / Z$ can be generated by $k$ elements.

Then there exists $M \leq G$ such that $[M, G, G]=1$ and $|G: M| \leq n^{k}$.

Proof. Let $M / Z=C_{G / Z}(T / Z)$. Then by Lemma $9,|G: M| \leq n^{k}$. Now $[T, M, G]=$ 1 , and in particular, $[T, M, T]=1$, so that $\left[T^{\prime}, M\right]=1$ by the Three Subgroup Lemma, and hence $\left[G^{\prime}, M\right]=1$. Now $[G, T, M] \leq\left[G^{\prime}, M\right]=1$. Applying the Three Subgroup Lemma again, we obtain that $[M, G, T]=1$. Consequently $[M, G] \leq$ $G^{\prime} \cap \mathbf{Z}(T)=Z$, and thus $[M, G, G]=1$.

Remark 13. The statement of Lemma 12 is also true if $n$ and $k$ are infinite cardinals.

Lemma 14. Let $G$ be a finite group and $\left|G^{\prime}: Z\right|=n$. Then there exists $T$ as in Lemma 12 with $k \leq 2 \log _{2} n$.

Proof. We define the elements $y_{i+1}, z_{i+1} \quad(0 \leq i \leq l-1)$ recursively by applying Lemma 11 for $V_{i}=\left\langle Z,\left[y_{1}, z_{1}\right],\left[y_{2}, z_{2}\right], \ldots,\left[y_{i}, z_{i}\right]\right\rangle$ and $U_{i}=C_{G^{\prime}}\left(V_{i}\right)$. Now we have that

and

$$
Z=V_{0} \leq V_{1} \leq V_{2} \leq \cdots \leq V_{l}=G^{\prime}
$$

$$
G^{\prime}=U_{0} \geq U_{1} \geq U_{2} \geq \cdots \geq U_{l}=Z
$$

where $l$ is the smallest integer such that $V_{l}=G^{\prime}$ and $U_{l}=Z$. It is clear that $l \leq \log _{2} n$. Now $T=\left\langle Z, y_{1}, z_{1}, y_{2}, z_{2}, \cdots, y_{l}, z_{l}\right\rangle$ has the required properties. 
Proof of Theorem 1. It follows immediately from Lemma 12 and Lemma 14 that there exists a subgroup $M$ of $G$ such that $|G: M| \leq n^{2 \log _{2} n}$ and $M \leq \mathbf{Z}_{\mathbf{2}}(G)$.

Proof of Theorem 4. First, we choose a subgroup $T_{1}$ such that $T_{1}^{\prime} Z=G^{\prime}$ and $\left|T_{1}\right| \leq$ $\kappa$. Let $Q$ be a coset representative system for $Z$ in $G^{\prime} \backslash \mathbf{Z}(G)$. We choose elements $y_{q}$ for all $q \in Q$ such that $y_{q} \notin C_{G}(q)$. The set $T_{2}=\left\{y_{q} \mid q \in Q\right\}$ has cardinality $\kappa$ and clearly $C_{G^{\prime}}(Y)=Z$. Let $T=\left\langle T_{1}, T_{2}\right\rangle$. Then $|T|=\kappa$, and the same argument as in Lemma 12 completes the proof.

\section{Groups With CyClic Derived subgroups}

In this section we focus our attention on groups with cyclic derived subgroups.

Lemma 15. Let $G$ be a group, and write $Z=G^{\prime} \cap \mathbf{Z}(G)$. Assume that $G^{\prime}$ is a p-group and $G^{\prime} / Z$ is cyclic of order $n$. Then there exists a subgroup $M \leq G$ such that $[M, G, G]=1$ and $|G: M| \leq n^{2}$.

Proof. Let $x \in G^{\prime}-Z$ such that $x^{p} \in \mathbf{Z}(G)$. Set $C=C_{G}(x)$. It follows that $C_{G}(y) \cap G^{\prime}=\mathbf{Z}(G) \cap G^{\prime}$ for all $y \in G-C$. Using Lemma 10 we can find $a \in G-C$ and $b \in G$ such that $\langle Z,[a, b]\rangle=G^{\prime}$. Let $T=\langle Z, a, b\rangle$, and note that $T$ satisfies the three conditions of Lemma 12 with $k=2$.

Proof of Theorem \%. We reduce to the case where $G^{\prime}$ is a $p$-group. For each prime divisor $p$ of $\left|G^{\prime}\right|$ let $N_{p}$ be the normal $p$-complement of $G^{\prime}$ and work in the factor group $G / N_{p}$. This factor group satisfies the hypotheses with $n$ replaced by a divisor of $n_{p}$, the $p$-part of $n$. Using the preceding lemma, we know that there exists a subgroup $M_{p} \leq G$ such that $\left[M_{p}, G, G\right] \leq N_{p}$ and $\left|G: M_{p}\right| \leq\left(n_{p}\right)^{2}$. Let $M=\bigcap M_{p}$. Then $[M, G, G] \leq \bigcap N_{p}=1$ and $|G: M| \leq \prod\left(n_{p}\right)^{2}=n^{2}$.

Proof of Theorem 8. Using the multiplicativity of Euler's $\varphi$ function, as in the previous proof, we can reduce to the case where $G^{\prime}$ is a $p$-group. If $G^{\prime} \cap \mathbf{Z}(G)>1$, then by Theorem 7 , the index of the second center is at most $(n / p)^{2}<n \varphi(n)$. In the case of $p=2$ the unique element of order 2 in $G^{\prime}$ is central in $G$, thus $G^{\prime} \cap \mathbf{Z}(G)>1$. We can assume therefore that $G^{\prime} \cap \mathbf{Z}(G)=1$ and in particular $p>2$. Now let $D=C_{G}\left(G^{\prime}\right)$, and note that $[G, D, D]=1$. Therefore $D^{\prime} \leq \mathbf{Z}(G)$ by the Three Subgroup Lemma. Then $D^{\prime} \leq G^{\prime} \cap \mathbf{Z}(G)=1$; so $D$ is abelian. It is obvious that $G / D \leq \operatorname{Aut}\left(G^{\prime}\right)$. Since $G^{\prime}$ is a cyclic $p$-group and $p>2$, we have that $G / D$ is cyclic of order dividing $\varphi(n)$. If $x$ generates $G$ modulo $D$, let $C=C_{D}(x)$. Then $C$ centralizes $D\langle x\rangle=G$, and hence $C \leq \mathbf{Z}(G)$. Consequently $|D: C|=|[D, x]| \leq n$, and we deduce that $|G: \mathbf{Z}(G)| \leq|G: D||D: C| \leq n \varphi(n)$.

Remark 16. I. M. Isaacs [2] proved that if $H$ is a capable nilpotent group with cyclic derived subgroup and all elements of order 4 are central in $H$, then $\mid H$ : $\left.Z(H)|=| H^{\prime}\right|^{2}$. In this result the assumption about elements of order 4 cannot be omitted as the example of the dihedral group $D$ of order $2^{n}(n \geq 3)$ shows. It is a capable group, $D^{\prime}$ is a cyclic group of order $2^{n-2}$ and $|D: Z(D)|=2^{n-1}$.

\section{ACKNowledgments}

The authors are very grateful to P. P. Pálfy and L. Pyber and especially to the referee for helpful comments. 


\section{REFERENCES}

[1] H. Heineken, Nilpotent groups of class two that can appear as central quotient groups, Rend. Sem. Mat. Univ. Padova 84 (1990), 241-248. MR1101296 (92c:20068)

[2] I. M. Isaacs, Derived subgroups and centers of capable groups, Proc. Amer. Math. Soc. 129 (2001), 2853-2859. MR1840087 (2002c:20035)

[3] I. D. Macdonald, Some explicit bounds in groups with finite derived groups, Proc. London Math. Soc (3) 11 (1961), 23-56. MR0124433(23:A1745)

[4] K. Podoski, Groups covered by an infinite number of Abelian subgroups, Combinatorica 21 (3) (2001), 413-416. MR.1848059 (2002e:20061)

[5] K. Podoski, B. Szegedy, Bounds in groups with finite Abelian coverings or with finite derived groups, J. Group Theory 5 (2002), 443-452. MR1931369 (2003i:20052)

[6] Derek J. S. Robinson, A Course in the Theory of Groups, Springer-Verlag, New York (1982). MR0648604 (84k:20001)

[7] J. Wiegold, Multiplicators and groups with finite central factor-groups, Math. Z. 89 (1965), 345-347. MR0179262 (31:3510)

Department of Algebra and Number Theory, Eötvös University, Pázmány Péter SÉTÁNy 1/C, H-1117 BudAPEST, Hungary

E-mail address: pcharles@cs.elte.hu

Current address: Alfréd Rényi Institute of Mathematics, Hungarian Academy of Sciences, POB 127, H-1364 Budapest, Hungary

E-mail address: pcharles@renyi.hu

Alfréd Rényi Institute of Mathematics, Hungarian Academy of Sciences, POB 127, H-1364 Budapest, Hungary

E-mail address: szegedy@renyi.hu 